\title{
The molecular cloud and embedded young stellar population associated with IRAS 18236-1205
}

\author{
Ricardo Retes, Abraham Luna, Divakara Mayya and Luis Carrasco \\ Instituto Nacional de Astrofísica, Óptica y Electrónica (INAOE), Mexico
}

\begin{abstract}
We test a membership method to select embedded young stellar objects (YSOs) from a Galactic molecular cloud with ongoing massive star formation using multiband analysis. We select and discuss the embedded stellar population in the molecular cloud associated with IRAS 18235-1205, a small, geometrically well-defined Galactic molecular cloud. The IRAS source has infrared fluxes characteristic of an UCHII region, $\operatorname{CS}(J=2-1)$ emission, and methanol and water maser emission, suggesting that this region is a good candidate for studies of young, massive star formation. The selection method of embedded stellar populations is based on the spatial distribution of ${ }^{13} \mathrm{CO}(J=1-0)$ and Spitzer/MIPS $24 \mu \mathrm{m}$ point sources. Photometric analysis using near/mid-infrared images are used to test our selection criteria. Three objects are associated with the IRAS source; two have a characteristic spectral-energy distribution (SED) of a Class I/0 object (protostar) and the third has an SED of Class II.
\end{abstract}

Keywords. ISM: molecular clouds, stars: formation, stars: pre-main-sequence, infrared: stars

The full poster (in pdf format) is available at http://www.astro.iag.usp.br/ 〜iaus266/Posters/pRetes.pdf. 